\section{Measuring fibrosis without biopsies}

Kidney fibrosis is a common cause of declining renal function in patients with chronic kidney disease (CKD) but cannot currently be detected without a kidney biopsy. However, imaging techniques might provide a noninvasive alternative for the assessment of renal fibrosis, according to a new report by Peter Boor and colleagues.

"We focused on potential biomarkers that would allow for the monitoring of fibrosis and might serve as end points in clinical trials," explains Boor. The researchers showed that, in rodents and humans, the extracellular matrix protein elastin is not extensively expressed in healthy kidneys but is strongly upregulated in areas of fibrosis. The next step was to test an elastin-specific MRI agent, ESMA, which significantly accumulated in mouse fibrotic kidneys but not in healthy tissue. After incubation with ESMA, human biopsy samples of fibrotic kidneys also had a higher ESMA signal than samples from healthy kidneys. Techniques such as western blot and immunofluorescence verified elastin expression and the presence of renal fibrosis; in vivo and ex vivo competition experiments validated the specificity of the ESMA signal.

The researchers then used imaging with ESMA to monitor the progression of fibrosis in the adenine-induced nephropathy mouse model and demonstrated a progressive increase in the ESMA signal over 3 weeks. An analysis of the contrast-to-noise ratio indicated that the ESMA signal peaked at $24 \mathrm{~h}$ but was mostly cleared from the kidney after $72 \mathrm{~h}$. Moreover, in two mouse models of fibrosis, mice treated with drugs that reduce fibrosis had significantly less elastin deposition than untreated mice (verified by histology), and this therapeutic response could be quantified by noninvasive imaging with ESMA. Last, in a model of reversible adenine nephropathy in which mice were allowed to recover after an initial injury, the ESMA imaging approach detected persistent fibrotic damage despite apparent normal kidney function according to serum markers.

"Our study lays the foundations for the translation of this diagnostic technique to patients with CKD," concludes Boor.

\section{Monica Wang}

ORIGINAL ARTICLE Sun, Q. et al. Elastin imaging enables noninvasive staging and treatment monitoring of kidney fibrosis. Sci. Transl Med. https://doi.org/10.1126/scitranslmed. aat4865 (2019)

\title{
A novel SGLT1-mediated mechanism of hyperglycaemia-induced hyperfiltration
}

Sodium-glucose cotransporter 2 (SGLT2) is thought to have a role in the pathogenesis of glomerular hyperfiltration in diabetes mellitus. A recent study by Jie Zhang and colleagues reports a novel mechanism by which SGLT1 might also contribute to the control of tubuloglomerular feedback (TGF) and glomerular filtration rate (GFR) in the setting of hyperglycaemia.

The researchers report that intravenous injection of glucose induced hyperglycaemia and significantly increased GFR in nondiabetic mice. Using microperfusion and micropuncture techniques, they show that tubular glucose inhibits the TGF response in vitro and in vivo and stimulates the generation of nitric oxide $(\mathrm{NO})$ at the macula densa. As these effects were blocked by an SGLT1 inhibitor and further analyses indicated that SGLT1 is expressed in macula densa cells, the researchers suggest that tubular glucose inhibits TGF and stimulates NO generation via SGLT1.

Zhang and colleagues also show that glucose upregulates the expression and phosphorylation of nitric oxide synthase 1 (NOS1) in human and mouse renal cortex. Selective knockout of NOS1 in macula densa cells abrogated glucoseinduced NO generation, inhibition of the TGF response and hyperfiltration in nondiabetic mice.

"We assume that hyperglycaemia increases glucose filtration and enhances SGLT2mediated sodium-glucose reabsorption, which decreases $\mathrm{NaCl}$ delivery to the macula densa and inhibits the vasoconstrictor TGF tone," comments Zhang. "Our study suggests that increased luminal glucose at the macula densa is also sensed by SGLT1, which upregulates NOS1 expression and activity and further reduces vasoconstrictor TGF tone. Thus, these two signalling pathways additively promote glomerular hyperfiltration in diabetes."

Ellen F. Carney

ORIGINAL ARTICLE Zhang, J. et al. Macula densa SGLT1NOS1-tubuloglomerular feedback pathway, a new mechanism for glomerular hyperfiltration during hyperglycemia. J. Am. Soc. Nephrol. https://doi.org/10.1681/ASN.2018080844 (2019)

\section{RENAL CARCINOMA}

\section{Unique metabolic traits identify CCPAP}

Renal cell carcinoma (RCC) is often caused by genetic mutations that disrupt cellular metabolism. In clear cell papillary RCC (CCPAP), driver mutations are absent but mitochondrial changes still result in a unique metabolic profile, according to a new report by Ed Reznik, Costas Lyssiotis, Satish Tickoo, Ari Hakimi and colleagues.

The researchers compared metabolite abundance in CCPAP lesions, adjacent healthy renal tissue and clear cell RCC (ccRCC) lesions, and found that polyol pathway metabolites, including sorbitol and fructose, were elevated in CCPAP, as was the NADH/ NAD ratio. By contrast, metabolites involved in upper glycolysis, usually high in ccRCC, were present at normal levels in CCPAP. Similarly to other RCC histologies, high levels of glutathione implied a cellular oxidative stress response in CCPAP tumours.

Malignant cells often downregulate genes linked to oxidative phosphorylation and upregulate hypoxia-related genes, including HIF. This pattern of gene expression was also observed in CCPAP tumours, but normal lactate levels suggested that mitochondria were not reprogrammed to favour glycolysis.

"Unlike related RCC histologies, HIF activation in CCPAP tumours occurs in the absence of inactivation of $\mathrm{VHL}$ and TCEB1," remarks Reznik. In fact, genomic profiling of CCPAP tumours showed that mutational burden was low and that copy number alterations and genomic rearrangements were absent. "The only recurrent genotype in CCPAP was mitochondrial DNA depletion, which might induce sorbitol accumulation by disrupting mitochondrial oxidative phosphorylation," explains Lyssiotis.

"Mitochondrial dysfunction may be one of the key initiating events in CCPAP," suggests Tickoo. "Our findings argue for orthogonal profiling of the large cross-section of cancers that lack identifiable driver mutations," adds Hakimi. "This enhanced profiling might involve metabolomic, epigenomic and proteomic parameters."

Monica Wang

ORIGINAL ARTICLE Xu, J. et al. Abnormal oxidative metabolism in a quiet genomic background underlies clear cell papillary renal cell carcinoma. eLife https://doi.org/ 10.7554/eLife.38986 (2019) 